\title{
ESCALA DE CURIOSIDADE RELACIONADA AO TRABALHO: VALIDAÇÃO E APLICAÇÃO NO CONTEXTO BRASILEIRO
}

\section{WORK-RELATED CURIOSITY SCALE: VALIDATION AND APPLICATION IN THE BRAZILIAN CONTEXT}

\section{ROMULO MATOS DE MORAES}

Diretor-Geral - IFES Campus de Alegre

Doutorando em Cognição e Linguagem - Universidade Estadual do Norte Fluminense (UENF) Orcid: https://orcid.org/0000-0003-2357-0869 / E-mail: romuloifes@gmail.com Rodovia BR 482, Km 47, Rive, Alegre-ES

\section{ROSALEE SANTOS CRESPO ISTOE}

Docente do PPG em Cognição e Linguagem - Universidade Estadual do Norte Fluminense (UENF) Doutora em Ciências pelo Instituto Fernandes Figueira - FIOCRUZ/RJ.

Orcid: 0000-0001-8959-9280 / E-mail: rosaleeistoe@gmail.com

Submissão: 10/06/2020. Revisão: 29/12/2020. Aceite: 16/04/2021. Publicação: 01/02/2022. DOI: http://dx.doi.org/10.22277/rgo.v15i1.6355

\section{RESUMO}

Objetivo: Introduzir no contexto brasileiro o conceito de Curiosidade Relacionada ao Trabalho, um estado em que o indivíduo tem interesse na forma como sua atuação afeta a empresa, confrontando problemas complexos a partir de novas estratégias.

Método: Estudo psicométrico com uso de Análise Paralela para validar a escala de mensuração do novo construto e utilização de Regressão Múltipla Linear para identificar sua relação com a performance laboral. A amostra contou com dados de 273 profissionais de vendas.

Principais resultados: Disponibilização de uma escala de mensuração de curiosidade replicável, metodologicamente adaptada e validada para o português. Identificou-se, no desempenho dos profissionais entrevistados, indícios consistentes de um impacto positivo e significativo dessa motivação intrínseca que leva a comportamentos investigativos.

Contribuições: Este trabalho contribui para o campo do Comportamento Organizacional ao possibilitar, para o cenário estudado, a inclusão da curiosidade como uma variável inovadora para fins de recrutamento, avaliações ou desenvolvimento de recursos humanos. O novo conceito pode auxiliar na explicação de variações de desempenho não captadas por construtos tradicionais.

Originalidade: A inserção de um conceito inédito no panorama brasileiro amplia as possibilidades de pesquisas organizacionais relacionadas ao comportamento e obtenção de conhecimento no ambiente de trabalho, além de permitir a comparação de resultados e troca de informações com a comunidade científica internacional.

Palavras-chave: Curiosidade Relacionada ao Trabalho. Desempenho Comercial. Psicometria.

Este é um artigo publicado em acesso aberto (Open Access) sob a licença Creative Commons Attribution, que permite uso, distribuição e reprodução em qualquer meio, sem restrições desde que o trabalho original seja corretamente citado. 


\begin{abstract}
Purpose: To introduce in the Brazilian context the concept of Work-Related Curiosity, a state in which the individual is interested in how his performance affects the company, confronting complex problems based on new strategies.
\end{abstract}

Method: Psychometric study using Parallel Analysis to validate a measurement scale for the new construct and Multiple Linear Regression to identify its relationship with work performance. The sample included data from 273 sales professionals.

Main findings: The present paper provides a curiosity measurement scale, methodologically adapted, and validated for Portuguese. Results from the regression revealed, among the interviewed professionals, evidence of a positive and significant impact of this intrinsic motivation that leads to investigative behaviors.

Contributions: This work contributes for the field of Positive Organizational Behavior enabling, in the studied scenario, the inclusion of curiosity as an innovative variable for the purposes of recruitment, evaluations or human resource development. The new concept can assist in explaining performance variations not captured by traditional constructs.

Originality: The insertion of an unprecedented concept in the Brazilian panorama expands the possibilities of organizational research related to behavior and obtaining knowledge in the work environment, it also allows the comparison of results and exchange of information with the international scientific community.

Keywords: Work-related Curiosity. Commercial Performance. Psychometrics.

\title{
1 INTRODUÇÃO
}

O constante fluxo de mudanças nas economias e, por consequência, nos mercados de trabalho demanda das empresas e seus colaboradores uma recorrente ressignificação de competências e adaptação frente à competição cotidiana (Rosa, 2013). Para ampliar o conhecimento científico e trazer respostas a tais demandas, novos conceitos são introduzidos às pesquisas organizacionais com o intuito de captar diferentes parcelas do amplo espectro do comportamento laboral. Esta pesquisa discute à luz do mundo do trabalho o conceito de curiosidade enquanto um comportamento adjacente às tendências comportamentais relacionadas à aquisição de conhecimento e aprendizagem, tão necessárias à sobrevivência das organizações.

Estudos seminais no campo da psicologia exploram o conceito de curiosidade como um fator crítico ao desenvolvimento da humanidade, na busca de informação e experiências, na persistência de tarefas desafiadoras ou ainda na exploração do ambiente (e.g. Peters, 1978; Zuckerman, 1994). As concepções teóricas sobre a curiosidade epistêmica estão presentes em várias áreas da investigação científica e partem inicialmente da lacuna entre o que se sabe e o que se quer saber (Loewenstein, 1994). Dessa forma, evoluem para questões como a propensão para resolver problemas e enigmas intelectuais, o incremento autoestimulado da criatividade e a tendência em elaborar hipóteses para obter conhecimento (Collins et al., 2004; Gross et al., 2020).

A psicologia tem investigado como a curiosidade influencia o comportamento e opera em propriedades situacionais diversificadas. Em um sentido geral observou-se que quando confrontadas com novas situações as pessoas que apresentaram maiores níveis de curiosidade adaptaram-se melhor e de forma menos estressante (Pulakos et al.,2000). 
Percebeu-se também que o fruto da curiosidade ultrapassa a aquisição de conhecimento e que seus traços se relacionam à propensão em explorar e conquistar situações novas e complexas. Sabe-se ainda que a curiosidade é geralmente uma experiência agradável e intensa que enseja a busca de interesses pessoalmente significativos. O prazer de descobrir novas perspectivas é um fator impulsionador para atividades inovadoras e uma forma revitalizante de encarar as oportunidades (Celik et al., 2016; Gross et al., 2020).

Os estudos anteriormente mencionados revelam, de uma forma ampla, a característica intrinsecamente motivadora e proativa da curiosidade epistêmica. Surpreendentemente, a importância desse comportamento para o mundo laboral ainda é relativamente recente na academia (Mussel et al., 2011). Entretanto, já é possível apontar pesquisas contemporâneas indicando que a Curiosidade Relacionada ao Trabalho (CRT) reforça habilidades cognitivas básicas e se relaciona positivamente com inovação no trabalho, além preencher lacunas não explicadas pelos tradicionais preditores da performance dos trabalhadores (Celik et al., 2016; Mussel, 2013). No Brasil, estudos com esse conceito ainda são inexistentes, o que abre novas vias de investigação em estudos organizacionais.

Este artigo tem como objetivo a validação e aplicação de uma Escala de Curiosidade Relacionada ao Trabalho adaptada para o contexto brasileiro (ECRT-Br), baseada na German Work-Related Curiosity Scale (GWRCS), desenvolvida originalmente por Mussel et al. (2011). Para a consecução desse objetivo foi realizada uma rigorosa avaliação psicométrica do instrumento de medida. Como primeira utilização da ECRT-Br explorou-se a relação do novo construto frente a um indicador de desempenho, abrindo caminhos sobre como a curiosidade impacta na busca cotidiana por resultados.

Os participantes da pesquisa são profissionais de vendas, uma categoria altamente ligada à criação de valor para clientes e organizações (Zhang \& Glynn, 2015). A literatura aponta que os fatores de personalidade têm especial relevância nessa atividade quanto ao fortalecimento dos vínculos com clientes e formação de redes de relacionamento (Herjanto \& Franklin, 2019; Zhang \& Glynn, 2015; Bartkus et al., 2011), porém tais apontamentos são predominantemente oriundos de análises fundamentadas na tradicional estrutura "Big Five Personality" (Barrick \& Mount, 1991; Herjanto \& Franklin, 2019). Essa observação oportuniza a abordagem proposta neste artigo com um novo conceito em uma cultura diferente dos estudos anteriores.

Este trabalho contribui para campo do Comportamento Organizacional Positivo (Luthans \& Youssef, 2007) ao disponibilizar um instrumento inédito de medida de CRT metodologicamente validado para o contexto nacional, além de fornecer indícios preliminares da forma como o conceito se relaciona com a performance laboral, suscitando novos caminhos de investigação para estudos futuros. Suas aplicações práticas posicionam a CRT como uma nova variável para fins de recrutamento, avaliações ou desenvolvimento de recursos humanos.

\section{REVISÃO DA LITERATURA}

\subsection{DEFININDO A CURIOSIDADE RELACIONADA AO TRABALHO}

A conceituação de Curiosidade Relacionada ao Trabalho (CRT) utilizada na fundamentação da escala proposta neste estudo refere-se à curiosidade epistêmica e inclui busca de informação, aquisição de conhecimento, aprendizagem e pensamento - aspectos considerados importantes no ambiente de trabalho (Mussel et al., 2011). Essa delimitação é importante para evitar a sobreposição ao conceito tradicional de curiosidade perceptiva, 
ligado à motivação que os seres vivos têm para buscar novos estímulos ou explorar o ambiente (Reeve, 2014).

A curiosidade epistêmica alude não apenas à busca por conhecimento e novidades, mas também à capacidade de dissipar incertezas, ambiguidades e complexidades, o que normalmente está associado a uma percepção positiva (Reeve, 2014; Compton \& Hoffman, 2019). Entretanto, é relevante ressaltar que esses comportamentos de curiosidade também podem se relacionar a sentimentos desagradáveis quando não se consegue a resposta procurada, o que leva a uma sensação de privação que cessa apenas quando o problema é resolvido ou ao se obter a resposta (Litmam, 2008).

Dentre os componentes da rede nomológica da curiosidade epistêmica, alguns relacionamentos a outros construtos merecem destaque dentro do contexto deste estudo, como a necessidade de cognição, envolvimento intelectual, motivação intrínseca para aprender, abertura para ideias, persistência e diligência (Amabile et al., 1994; Mussel, 2010).

Indivíduos dotados de curiosidade adaptam-se facilmente e apresentam comportamento proativo. Possuir adaptabilidade remete à capacidade de lidar com mudanças e responder a novos desafios e papéis no ambiente de trabalho (Griffin et al., 2007). O modelo atual de mercado está constantemente sujeito a fusões, reestruturações, introdução de novas tecnologias e redesenho do trabalho, ou seja, casos típicos de incerteza e interdependência. Alguns pontos cruciais para lidar com este cenário são adquirir novas habilidades e se estruturar para enfrentar mudanças na organização (Pulakos et al., 2000), características proeminentes entre os que possuem altos níveis de curiosidade (Gross et al., 2020; Mussel, 2010; Collins et al., 2004).

Entre os mais curiosos, o efeito estressor provocado por novas situações tende a ser menos percebido como negativo e mais como uma oportunidade efetiva de aprendizado (Pulakos et al., 2000; Mussel et al., 2011). Quanto à proatividade, uma relação significativamente positiva com a curiosidade foi identificada por Seaton e Beaumont (2008), reforçando a importância dessa característica para as demandas do mercado de trabalho. Parte disso ocorre em função de um interesse não só na resolução de problemas, mas também no conhecimento subjacente que envolve o esforço em desvendar o que é apresentado, surgindo assim resultados inovadores (Mussel et al., 2011; Celik et al., 2016).

A Curiosidade Relacionada ao Trabalho é assim um estado em que o indivíduo tem interesse na forma como sua atuação afeta a empresa, confrontando problemas complexos a partir de novas estratégias, em um fluxo que envolve prazer em aprender juntamente com um envolvimento intrínseco e inovador frente ao problema em si. É possível situar este construto no campo do Comportamento Organizacional Positivo (Luthans \& Youssef, 2007) que por influência da Psicologia Positiva (Seligman, 2004) propõe menos foco no negativo/problemático de forma a canalizar e valorizar os aspectos edificantes dos indivíduos na busca de resultados éticos, produtivos e sustentáveis.

\subsection{FATORES PESSOAIS E DESEMPENHO}

As empresas comerciais têm uma carga de exigência crescente de seus vendedores, demandando que lidem com situações cada vez mais complexas, persuadam os compradores em ambientes altamente competitivos, construam confiança e alcancem as metas organizacionais de vendas (Franklin \& Marshall, 2019). A utilização eficaz do capital humano dos funcionários no local de trabalho e seu nível de envolvimento cognitivo e emocional têm se mostrado como fatores-chave para o sucesso organizacional (Kotze, 2018). 
Dada a importância do desempenho dos vendedores, muitos estudiosos têm se concentrado em identificar um amplo espectro de componentes que afetam o resultado desses profissionais (Evans et al., 2012) como, por exemplo, fatores pessoais, organizacionais, sociais e de mercado. Dentre esses fatores, a dimensão pessoal é a mais investigada, provavelmente por ser a mais complexa e dinâmica, além de suscitar questões sobre como diferentes traços de personalidade afetam a performance de vendas (Herjanto \& Franklin, 2019). Sugere-se que os fatores pessoais não são apenas nichos de pesquisa amplamente explorados, mas ingredientes-chave na construção de relacionamentos com os stakeholders (Zhang \& Glynn, 2015).

Partindo dessa discussão, no presente estudo optou-se por uma amostra de vendedores devido à possibilidade de utilizar nas análises um indicador de desempenho tangível, proveniente de equipes de vendas, para verificar como a CRT afeta os resultados organizacionais.

\section{ASPECTOS METODOLÓGICOS}

Pesquisa quantitativa, descritiva, com coleta de dados primários e de corte transversal, realizada com profissionais de vendas vinculados a um distribuidor de produtos de grandes marcas multinacionais nos segmentos de higiene, perfumaria e alimentos industrializados. A amostragem foi não probabilística por conveniência, selecionada de acordo com o acesso dos pesquisadores e devido e à falta de dados precisos quanto ao número de vendedores que atuam no Brasil. Optou-se por essa população em função de sua relação intrínseca com o alcance de metas, permitindo assim incluir o desempenho como uma variável mensurável, ligada ao mundo do trabalho e passível de ser relacionada com a curiosidade nas análises estatísticas, o que possibilitou verificar de forma objetiva os primeiros resultados da nova escala no contexto laboral brasileiro.

A estratégia de análise dos dados foi composta de duas etapas: para a validação psicométrica da escala utilizou-se Análise Fatorial Exploratória (AFE) com técnica de Análise Paralela (Timmerman \& Lorenzo-Seva, 2011) e, para verificar a influência relativa da CRT no desempenho, utilizou-se uma Regressão Linear Múltipla.

\subsection{COLETA DE DADOS E CARACTERÍSTICAS DA AMOSTRA}

A empresa na qual os entrevistados trabalham apoiou os pesquisadores fornecendo os e-mails da equipe de vendas. Dentre os 355 profissionais convidados, obteve-se 273 respostas válidas, o que representa uma taxa de resposta de 76,9\%. A coleta de dados ocorreu ao longo do mês de setembro de 2020, de forma eletrônica por meio de um link para o formulário hospedado na plataforma Google Forms. O link, incorporado ao e-mail de convite, poderia ser aberto tanto em computadores desktop como em dispositivos móveis.

No preâmbulo do questionário foi apresentado um Termo de Consentimento Livre e Esclarecido, salientando que a participação era voluntária, anônima e que as informações prestadas seriam tratadas estatisticamente dentro de um contexto científico, sendo necessário o respondente assinalar sua concordância em campo próprio ou abandonar a página, sem nenhum prejuízo.

Os entrevistados atuam em dois estados do sudeste Brasileiro. Características demográficas: $61,5 \%$ do gênero masculino; tempo de médio de empresa cinco anos; $71,1 \%$ possui curso superior e destes, 7,7 \% possui pós-graduação. A idade média foi de 32 anos, variando de 19 a 54 anos, porém $80 \%$ dos respondentes estão concentrados na faixa de até 
Escala de curiosidade relacionada ao trabalho: validação e aplicação no contexto brasileiro

40 anos. Esses vendedores desenvolvem suas atividades presencialmente na maioria dos casos, mas informaram que aproximadamente $30 \%$ dos negócios ocorrem por telefone ou aplicativos de mensagens.

\subsection{MEDIDAS}

Escala de Curiosidade Relacionada ao Trabalho (ECRT-Br): o instrumento original denominado German Work-Related Curiosity Scale (GWRCS) foi desenvolvido por Mussel et al. (2011) e concebido a partir de uma perspectiva interacionista, onde padrões comportamentais estáveis estão vinculados a condições situacionais específicas. Seguiram-se ainda recomendações mais recentes em que se destaca a maior assertividade e aplicabilidade de avaliações de personalidade contextualizadas (Wright \& Mischel, 1987; Heggestad \& Gordon, 2008).

A escala original é unidimensional e conta com dez itens desenvolvidos e avaliados por especialistas com os seguintes critérios: os itens deveriam ser relacionados ao trabalho; se uma relação explícita de trabalho não fosse possível, o item deveria, pelo menos, tocar em comportamentos que são relevantes no local de trabalho (por exemplo, "Continuo buscando informações até conseguir entender questões complexas"). Além disso, os itens deveriam ser restritos a avaliar as preferências por certos comportamentos, o que significa que as autoavaliações de curiosidade ou eventos da vida pessoal foram omitidos. Os critérios para seleção de itens foram: alto poder discriminatório, alta validade convergente com abertura para experiência, bem como validade de conteúdo, com base no julgamento dos especialistas. Esses critérios foram escolhidos para desenvolver uma escala curta, confiável e válida para a avaliação da curiosidade relacionada ao trabalho (Mussel et al., 2011). Os escores dos itens variam de 1 ("Discordo totalmente") a 7 ("Concordo totalmente").

Verificou-se a inexistência de uma validação do instrumento de medida de Mussel et al. (2011), para o contexto brasileiro. Para esta constatação foram realizadas buscas nos indexadores SPELL, SciELO, Google Scholar, Sumarios.org e Portal de Periódicos CAPES/MEC com os descritores Escala de Curiosidade, Escala Curiosidade Relacionada ao Trabalho, Curiosidade e escala. O período da busca abrangeu o período de 2011, data da criação da escala validada, até março de 2021. Durante este levantamento, foi possível verificar ainda a escassez de investigações voltadas à curiosidade relacionada ao trabalho sejam qualitativas ou quantitativas.

Desempenho: para medir o desempenho laboral do entrevistado, foi solicitado que o respondente informasse o percentual de alcance de sua meta de vendas no mês anterior. Os valores variaram de 59 a 106\%, com uma média de $82,3 \%$. A opção por esta variável ocorreu por se tratar de um indicador tipicamente relacionado ao trabalho dos vendedores e com uma clara possibilidade de aferição numérica.

Variáveis de Controle: idade, tempo de empresa, nível de escolaridade e gênero. A inclusão ocorreu devido a suas possíveis covariações com as medidas focadas no estudo.

\subsection{TRADUÇÃO}

Apesar de originalmente criada no idioma alemão, os autores da GWRCS apresentaram na publicação do estudo sua versão para o inglês, da qual realizou-se a tradução e adaptação para o português. A primeira etapa de tradução foi conduzida por um tradutor independente e passou por um pré-teste com 12 alunos, dois professores do último período de 
Administração de Empresas, e um professor de Psicologia. O intuito foi verificar a clareza e o entendimento das afirmativas do questionário, no qual havia campos para sugestões e dúvidas. Este procedimento visa aprimorar os conteúdos, conforme recomendado por Ornstein (2013).

Entre os estudantes voluntários para o pré-teste, quatro atuavam em vendas no comércio, três em atividades administrativas e um no setor de tecnologia, os demais não possuíam outras atividades além das acadêmicas. A escala original tem caráter generalista e foi aplicada em respondentes de áreas diversas como setor financeiro, indústria automotiva, representantes de vendas, e-commerce e Tecnologia da Informação, além de estudantes universitários (Celik et al., 2016; Mussel, 2013). Como o instrumento possui uma proposta de utilização universal, sem assertivas provenientes de terminologias específicas de uma área, o pré-teste não foi limitado apenas ao público alvo da coleta de dados (Ornstein, 2013).

Posteriormente, um segundo tradutor especializado acompanhado dos pesquisadores revisou os itens da escala, observando equivalência semântica, adequação de expressões idiomáticas, equivalência cultural e equivalência conceitual. Conforme recomendado por Van De Vijver e Hambleton (1996), uniu-se o conhecimento proveniente da literatura específica ao tema à competência linguística dos tradutores, evitando-se que uma versão muito literal perdesse conteúdo. Finalizado este processo, pesquisadores, tradutores, o professor de psicologia convidado e a professora de língua portuguesa, reuniram-se para avaliar a versão final. Com exceção de pequenos ajustes gramaticais, a única alteração significativa aponta da pelo grupo foi no item 3 da escala, na qual a afirmativa: "Em relação aos problemas práticos, também estou interessado na teoria ligada ao assunto", foi substituída por: "Em relação a problemas práticos, também estou interessado nos conceitos que existem por trás da situação", assim, buscando uma assertiva melhor interpretável em situações práticas do mundo do trabalho. $O$ mesmo item também sofreu alteração na primeira etapa na qual a expressão era "teoria subjacente".

\section{APRESENTAÇÃO E DISCUSSÃO DOS RESULTADOS}

\subsection{VALIDAÇÃO DA ECRT-BR}

Por se tratar de um instrumento relativamente novo e com certa escassez de estudos, optou-se por uma nova AFE para confirmar se a retenção dos itens permanece a mesma na versão em português. Essa opção também é particularmente apropriada quando há pouca base teórica para especificar a priori o número e os padrões de fatores comuns (Hurley et al., 1997). Entretanto, mesmo sem a condução de uma Análise Fatorial Confirmatória (AFC), no mesmo processo também foram avaliados os principais índices de ajuste do modelo, uma vez que se optou por utilizar o software Factor (Lorenzo-Seva \& Ferrando, 2013) que possibilita obter essa informação juntamente com a AFE.

Conforme apresentado na tabela 1 , os dados são adequados à análise fatorial já que o Teste KMO foi superior a 0,80 e o teste de esfericidade de Barlett foi estatisticamente significante (Hair et al., 2009). A matriz do banco de dados foi analisada por correlação policórica, o método de extração de fator foi o Robust Diagonally Weighted Least Squares (RDWLS). 
Tabela 1

Adequação da matriz de correlação policórica

\begin{tabular}{lr}
\hline Descrição & Valor obtido \\
\hline Determinante da matriz & 0,008297408 \\
Teste de esfericidade de Bartlett & 1283,4 (df=45; P = 0,000010) \\
Medida Kaiser-Meyer-Olkin (KMO) & 0.9203 \\
BC* Bootstrap 95\% IC** do KMO & $(0.917-0.943)$ \\
*bias-corrected (correção de viés) ** intervalo de confiança. & \\
\hline
\end{tabular}

A técnica de retenção de fatorial foi a implementação otimizada de Análise Paralela, desenvolvida por Timmerman e Lorenzo-Seva (2011). Trata-se de um dos métodos mais precisos para determinar a retenção de fatores. Para sua realização o processo contou com 500 permutações aleatórias dos valores amostrais. Observando-se a Figura 1, é possível verificar que apenas um fator teve o percentual de variância explicada real (64,77\%) maior que a dos bancos de dados randômico $(24,23 \%)$ gerado no processo de reamostragem (boostrapping).

Figura 1

Retenção de fatores segundo \% variância explicada - real x simulada

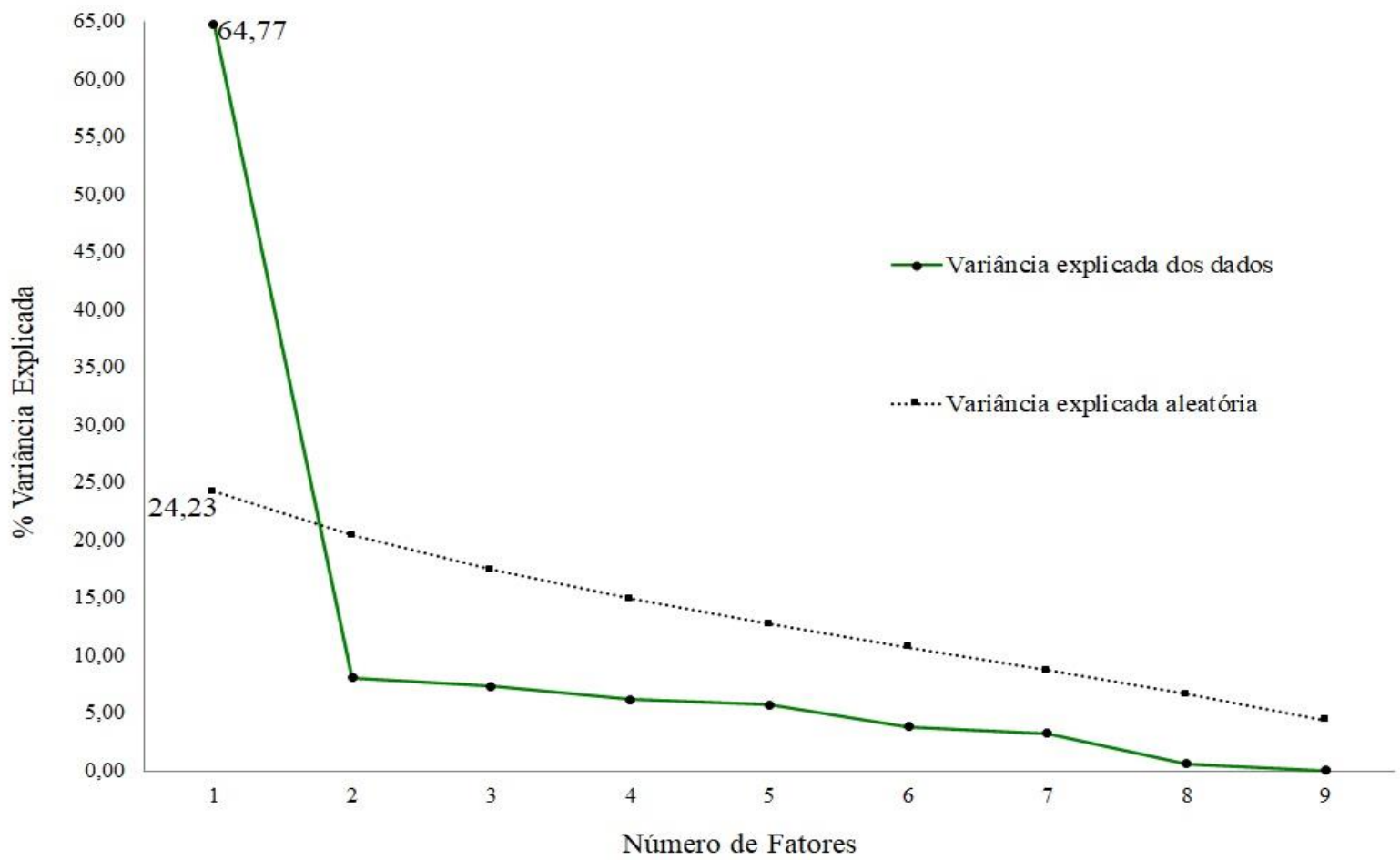

Além da Análise Paralela, a unidimensionalidade do instrumento também foi verificada pela técnica de aproximação de Ferrando e Lorenzo-Seva (2018). Conforme reportado na tabela 2, os valores dos indicadores UniCo, ECV e MIREAL atendem aos valores sugeridos pelos autores da técnica e confirmam que os dados podem ser tratados essencialmente como unidimensionais. 
Tabela 2

Aproximação da unidimensionalidade (avaliação geral)

\begin{tabular}{lrrr}
\hline \multicolumn{1}{c}{ Indicador } & Valor obtido & $\begin{array}{c}\text { BC Bootstrap } \\
\text { 95\% IC }\end{array}$ & $\begin{array}{c}\text { Valor } \\
\text { sugerido }\end{array}$ \\
\hline Congruência Unidimensional (UniCo) & 0,988 & $(0,979-0,995)$ & $>0,95$ \\
Variância comum explicada (ECV) & 0,908 & $(0,892-0,942)$ & $>0,85$ \\
Média residual das cargas absolutas dos itens (MIREAL) & 0,163 & $(0,134-0,175)$ & $<0,30$ \\
\hline
\end{tabular}

Os índices de ajuste encontrados na Tabela 3 apontam uma boa proporção de variância-covariância explicada (GFI - índice de qualidade de ajuste). Além disso, o índice de Tucker Lewis (TLI) indica independência entre as variáveis mensuradas, mesmo com uma amostra relativamente pequena, já que o índice de ajuste comparativo (CFI) apresentou-se dentro dos valores de ajustamento. O valor RMSEA (raiz da média dos quadrados dos erros de aproximação) obtido indica um excelente ajuste do modelo à população uma vez que se encontra próximo a 0,06 (Thompson, 2004).

Tabela 3

Índices de ajuste do modelo, consistência interna e replicabilidade

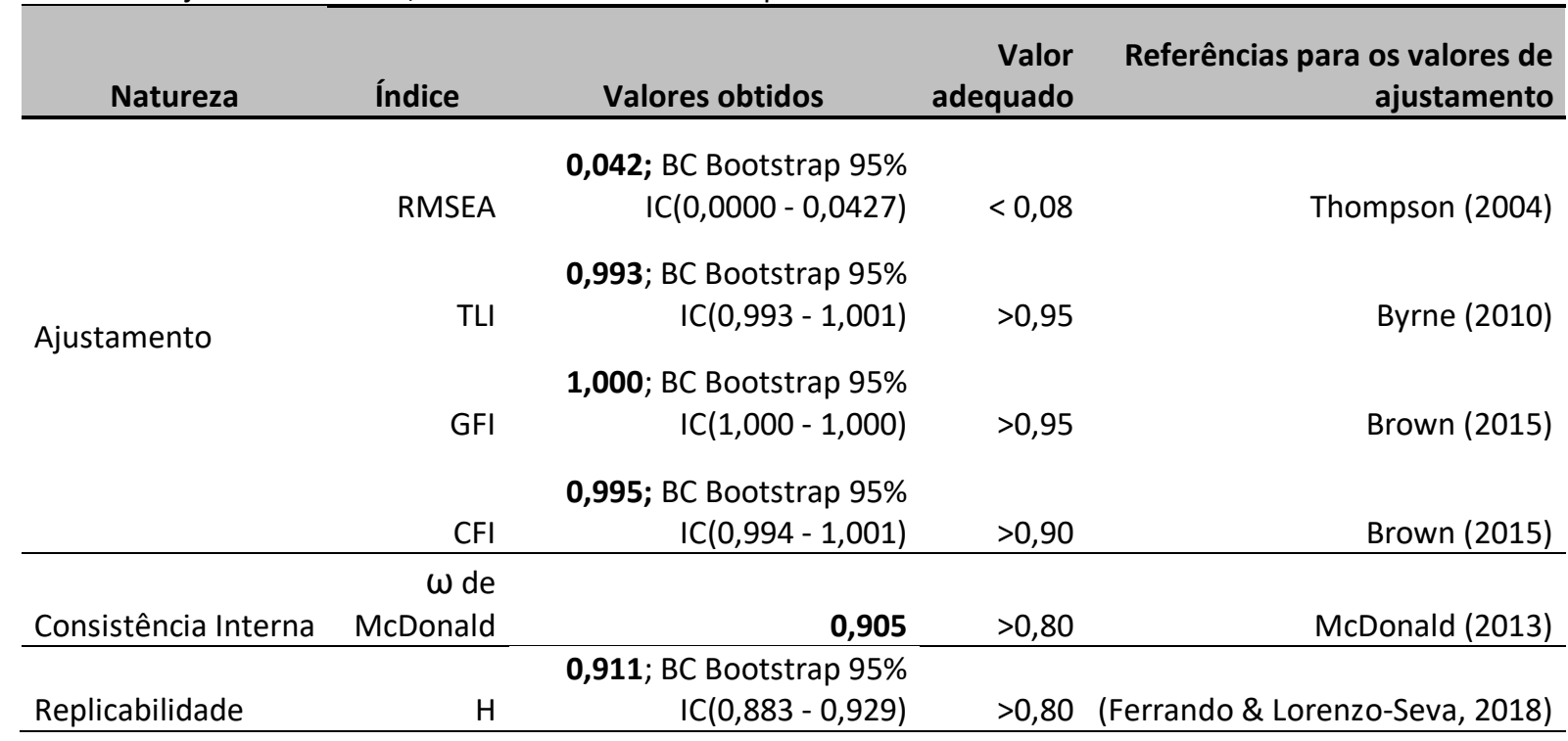

A tabela acima demonstra ainda que a escala deste estudo apresentou um ômega de McDonald de 0,905, indicando um índice de consistência interna extremamente satisfatório. Quanto à replicabilidade do construto, obteve-se um índice $\mathrm{H}$ maior que $0,80(\mathrm{H}=0,911 ; \mathrm{BC}$ Bootstrap 95\% IC 0,883 - 0,929), o que permite sugerir que a variável latente CRT tem probabilidade de permanecer estável em outros estudos (Ferrando \& Lorenzo-Seva, 2018). A versão definitiva com os dez itens da ECRT-Br e suas respectivas cargas fatoriais está apresentada na tabela 4. 
Escala de curiosidade relacionada ao trabalho: validação e aplicação no contexto brasileiro

Tabela 4

Versão em português dos itens da ECRT-Br com as cargas fatoriais

\begin{tabular}{clrr} 
Item & \multicolumn{1}{c}{ Descrição } & $\begin{array}{c}\text { Cargas } \\
\text { Fatoriais }\end{array}$ & $\begin{array}{c}\text { Bootstrapping } \\
\text { (95\% IC BCa) }\end{array}$ \\
\hline 1 & Estou interessado em saber como minha contribuição afeta a empresa. & 0,623 & $(0,482-0,714)$ \\
\hline 2 & Eu gosto de desenvolver novas estratégias. & 0,743 & $(0,654-0,809)$ \\
\hline & $\begin{array}{l}\text { Em relação a problemas práticos, também estou interessado nos } \\
\text { conceitos que existem por trás da situação. }\end{array}$ & 0,682 & $(0,591-0,753)$ \\
\hline & $\begin{array}{l}\text { Quando encontro problemas complexos, gosto de procurar novas } \\
\text { soluções. }\end{array}$ & 0,763 & $(0,698-0,818)$ \\
\hline 5 & Eu gosto de ponderar e pensar. & 0,718 & $(0,602-0,791)$ \\
\hline 6 & Estou ansioso para aprender. & 0,769 & $(0,703-0,824)$ \\
\hline 7 & Eu continuo pensando em um problema até resolvê-lo. & 0,614 & $(0,484-0,692)$ \\
\hline 8 & Eu questiono as práticas existentes. & 0,736 & $(0,659-0,801)$ \\
\hline & Continuo buscando informações até conseguir entender questões & 0,733 & $(0,636-0,792)$ \\
\hline 9 & complexas. & 0,638 & $(0,537-0,721)$ \\
\hline
\end{tabular}

Os resultados apresentados nesse capítulo permitem a aceitabilidade do modelo unidimensional com bons indícios de que a ECRT-Br possui características psicométricas adequadas de aferição e replicabilidade em investigações relacionadas ao mundo do trabalho. Em conformidade com a versão original, seus dez itens são contextualizados com situações relacionáveis ao trabalho (Mussel et al., 2011) envolvendo aquisição de novos conhecimentos, habilidades, ideias e soluções. Dessa forma, a carga conceitual da medida aqui validada contém ainda, de forma latente, a proatividade e o espírito inovador como componentes de sua estrutura (Celik et al., 2016; Mussel, 2013; Mussel et al. 2011).

\subsection{APLICAÇÃO DA ESCALA}

A interpretação dos resultados com o uso da ECRT-Br deve considerar que, quanto maior o escore médio do construto, maior a curiosidade relacionada ao trabalho do entrevistado naquele ambiente organizacional. Sugere-se classificar como alto um escore acima de 5; médio para valores entre 3,1 e 4,9; baixo para escores menores ou iguais a 3 . No presente estudo o conjunto das observações indicou uma CRT alta (média amostral de 5,12) com uma dispersão satisfatória $(D P=1,34)$. A tabela 5 traz as frequências por faixa de interpretação sugerida.

Tabela 5

Estatísticas descritivas para CRT

\begin{tabular}{|c|c|c|c|c|}
\hline \multirow[b]{2}{*}{ Descritivos } & \multicolumn{3}{|c|}{ Faixas obtidas } & \multirow{2}{*}{$\begin{array}{l}\text { Total da } \\
\text { amostra }\end{array}$} \\
\hline & $\begin{array}{l}\text { CRT Baixa } \\
\text { (média } \leq 3 \text { ) } \\
\end{array}$ & $\begin{array}{c}\text { CRT Média } \\
\text { (média entre } 3,1 \text { e 4,9) }\end{array}$ & $\begin{array}{l}\text { CRT Alta } \\
\text { (média } \geq 5 \text { ) }\end{array}$ & \\
\hline Frequência (N) & 41 & 16 & 216 & 273 \\
\hline Frequência (\%) & $15,02 \%$ & $5,86 \%$ & $79,12 \%$ & $100 \%$ \\
\hline Média (M) & 2,2 & 4,25 & 5,73 & 5,12 \\
\hline Desvio Padrão (DP) & 0,48 & 0,66 & 0,39 & 1,34 \\
\hline Intervalo & $1,2-3,0$ & $3,1-4,8$ & $5,0-7,0$ & $1,2-7,0$ \\
\hline
\end{tabular}


Para explorar o tipo de associação e a magnitude entre CRT (variável independente $\beta_{1} C R T$ ) e o desempenho no trabalho (variável dependente YDESEMPENHo), foi conduzida uma regressão múltipla, apresentada na Tabela 6 . A análise foi controlada pelas variáveis: Idade $\left(\beta_{2} I D A D E\right)$, Tempo de Empresa $\left(\beta_{3} T E\right)$, Gênero $\left(\beta_{4} G E N E R O\right)$ e Escolaridade $\left(\beta_{5} E S C O L\right)$, conforme o modelo apresentado na equação (1). O termo $\varepsilon$ representa o erro amostral e $\beta_{o}$ a constante de regressão.

$$
Y_{D E S E M P E N H O}=\beta_{O}+\beta_{1} C R T+\beta_{2} I D A D E+\beta_{3} T E+\beta_{4} G E N E R O+\beta_{5} E S C O L+\varepsilon
$$

Tabela 6

Resultado da regressão linear multivariada

\begin{tabular}{|c|c|c|c|c|}
\hline \multicolumn{5}{|c|}{ Resumo do Modelo } \\
\hline$R^{2}$ ajustado: 0,343 & & \multicolumn{3}{|c|}{ Erro padrão da estimativa: 6,681} \\
\hline Durbin-Watson: 2,02 & & \multicolumn{3}{|c|}{ ANOVA: $F(5,267)=29,380, p<0,001$} \\
\hline \multicolumn{5}{|c|}{ Coeficientes de Regressão } \\
\hline Variáveis Independentes & $\boldsymbol{\beta}$ (padronizado) & & $\mathrm{t}$ & FIV \\
\hline CRT & 0,620 & $* * *$ & 10,639 & 1,405 \\
\hline IDADE & $-0,045$ & ns & $-0,714$ & 1,619 \\
\hline TEMPO DE EMPRESA & 0,169 & $* *$ & 2,573 & 1,795 \\
\hline GÊNERO & $-0,043$ & ns & $-0,868$ & 1,032 \\
\hline ESCOLARIDADE & 0,033 & ns & 0,628 & 1,134 \\
\hline
\end{tabular}

O modelo apresentou uma boa adequação, não indicando multicolinearidade entre as variáveis independentes uma vez que o fator de inflação da variância (FIV) foi menor que cinco. Não foram identificados problemas de autocorrelação nos resíduos considerando um valor de Durbin-Watson próximo a dois (Hair et al., 2009).

A literatura aponta diversos preditores para o desempenho comercial, como mercado, economia, engajamento, experiência e fatores emocionais, dentre outros (Herjanto \& Franklin, 2019; Bartkus et al., 2011). Assim, permite-se admitir que o percentual de $34,3 \%$ explicação $\left(R^{2}\right)$ para o modelo é satisfatório na medida em são reconhecidos outros antecedentes para a variável dependente e que o objetivo almejado nessa etapa do estudo é explorar somente evidências da influência da CRT no desempenho.

$O$ coeficiente beta positivo e altamente significativo $(\beta=0,62 ; p<0,001)$ para a variável CRT fornece, para o contexto brasileiro, os primeiros indícios do potencial aumento de desempenho influenciado pela Curiosidade Relacionada ao Trabalho. Por consequência, sugere-se que a CRT, ou seja, a motivação intrínseca que leva a comportamentos investigativos ou exploratórios em áreas relacionadas ao trabalho pode ser incluída entre os preditores do desempenho de funcionários. Este achado reforça estudos anteriores que apontam que traços de personalidade têm propensão a impactar significativamente a aprendizagem no local de trabalho (Hassan et al., 2015) e, por consequência, na consecução de objetivos.

Esses resultados corroboram com Mussel (2013) quanto ao efeito da CRT na performance laboral e oferecem um suporte adicional para a validade do conceito e sua aplicabilidade. Como no presente estudo, a atividade da população analisada é pautada pela exigência quanto ao alcance de metas (Franklin \& Marshall, 2019), sugere-se que o interesse 
Escala de curiosidade relacionada ao trabalho: validação e aplicação no contexto brasileiro

por novidades, típica dos mais curiosos, é um recurso que permite, por exemplo, entender melhor as demandas dos clientes de forma proativa em situações em que há um natural grau de incerteza como é a representação comercial (Seaton \& Beaumont, 2008; Griffin et al., 2007; Evans et al., 2012).

Quanto às variáveis de controle, é possível apontar resultados complementares relevantes para o entendimento do conceito. $O$ tempo de empresa também apresentou uma associação positiva com o desempenho, um indicativo da importância da experiência na busca de resultados comerciais, porém com magnitude e nível de significância menor que os da curiosidade. A idade não teve impacto significativo no desempenho o que estabelece um contraponto a estudos que apontam um declínio de performance com a progressão etária (Alessandri et al., 2019; Bertolino et al., 2013). Da mesma forma gênero e escolaridade também não foram significantes na regressão. Estes indícios apontam caminhos para estudos a respeito do desempenho comercial sob a perspectiva da desconstrução dos estereótipos no local de trabalho.

\section{CONSIDERAÇÕES FINAIS}

O estudo disponibiliza uma escala de CRT metodologicamente traduzida e validada para o contexto nacional, assegurando que os aspectos de medição são fidedignos, mantendo a carga conceitual do estudo original, de forma a permitir sua replicabilidade, comparação de resultados e troca de conhecimentos com a comunidade científica internacional.

A estrutura do construto apresentada nesse artigo remete aos indivíduos que buscam novas estratégias para suas organizações, prospectam informações, se interessam nas questões adjacentes aos problemas do cotidiano e, de forma crítica, questionam e aprendem com tais situações, contribuindo assim para o desenvolvimento individual e da empresa.

Os resultados apresentados permitem sugerir que profissionais curiosos estão propensos a uma performance melhor e que a CRT pode trazer impactos mais significativos que fatores tradicionais como idade, tempo de empresa e escolaridade. Como implicações práticas para a mensuração da curiosidade laboral, sugere-se que acessar os níveis individuais dessa característica pode auxiliar em ações de aconselhamento de carreira, desenvolvimento de potenciais e processos de seleção.

A CRT pode complementar os conceitos tradicionalmente investigados em psicologia organizacional de forma a elucidar parcelas das variações de desempenho não captadas por construtos clássicos. Assim, como principal contribuição, tem-se a possibilidade de inclusão do construto CRT em estudos organizacionais em conjunto com outras medidas de comportamento, o que abre novos caminhos para aumentar o poder de explicação em modelos de pesquisa quantitativos com desenhos relacionais multivariados. Fatores positivos ligados à personalidade são cada vez mais relevantes para o enfrentamento dos obstáculos e para o sucesso profissional. Sob esse prisma, a ECRT-Br caracteriza-se como uma inovadora ferramenta para ações de Desenvolvimento de Recursos Humanos voltadas à identificação e ao fomento da curiosidade nos integrantes das equipes. Como contribuição metodológica destaca-se o uso de técnicas atuais e robustas na AFE, como a Análise Paralela e Aproximação da Unidimensionalidade (Ferrando \& Lorenzo-Seva, 2018), relativamente pouco utilizadas em relação aos métodos tradicionais.

A despeito das contribuições elencadas e objetivos atingidos, é importante destacar as principais limitações do estudo: a primeira refere-se à especificidade da amostra, limitada apenas aos profissionais de vendas, não permitindo generalização dos resultados; a outra 
remete à ausência de outras medidas de comportamento organizacional para testes comparativos. Em razão dessas lacunas algumas frentes de pesquisa podem ser sugeridas para estudos futuros: utilizar o instrumento de medida ECRT-Br em populações de diferentes áreas de atuação da iniciativa privada ou do setor público, visando identificar a relação da CRT com outros parâmetros de desempenho; investigar a relação do novo construto com medidas consagradas de Comportamento Organizacional, como por exemplo Satisfação no Trabalho, Burnout ou Engajamento no Trabalho, explorando assim não apenas as resultantes da curiosidade, mas também possíveis fatores que a antecedem e influenciam seus níveis. $O$ estigma de que o aumento da idade poderia influenciar negativamente o desempenho não se confirmou, sugerindo uma possível quebra de paradigma que abre caminhos para confirmação ou refutação em novos trabalhos com variações maiores nas faixas etárias.

\section{REFERÊNCIAS}

Alessandri, G., Truxillo, D. M., Tisak, J., Fagnani, C., \& Borgogni, L. (2019). Within-individual age-related trends, cycles, and event-driven changes in job performance: A careerspan perspective. Journal of Business and Psychology, 1-20. https://doi.org/10.1007/s10869-019-09645-8.

Amabile, T. M., Hill, K. G., Hennessey, B. A., \& Tighe, E. M. (1994). The Work Preference Inventory: assessing intrinsic and extrinsic motivational orientations. Journal of Personality and Social Psychology, 66(5), 950. http://people.uncw.edu/caropresoe/edn500/500fall07/amabile-etal-wpi-article.pdf.

Barrick, M. R., \& Mount, M. K. (1991). The big five personality dimensions and job performance: a meta-analysis. Personnel Psychology, 44(1), 1-26. https://doi.org/10.1111/j.1744-6570.1991.tb00688.x.

Bartkus, K. R., Howell, R. D., \& Haws, N. D. (2011). the type a behavior pattern and sales performance: a theoretical clarification. The Marketing Management Journal, 21, 115127. http://www.mmaglobal.org/publications/MMJ/MMJ-Issues/2011-Fall/MMJ-2011Fall-Vol21-Issue2-Bartkus-Howell-Haws-pp115-127.pdf.

Bertolino, M., Truxillo, D. M., \& Fraccaroli, F. (2013). Age effects on perceived personality and job performance. Journal of Managerial Psychology. https://doi.org/10.1108/JMP07-2013-0222.

Brown, T. A. (2015). Confirmatory factor analysis for applied research. New York: Guilford publications.

Byrne, B. M. (2010). Structural equation modeling with AMOS: basic concepts, applications, and programming. New York: Routledge.

Celik, P., Storme, M., Davila, A., \& Myszkowski, N. (2016). Work-related curiosity positively predicts worker innovation. Journal of Management Development. https://doi.org/10.1108/JMD-01-2016-0013. 
Collins, R. P., Litman, J. A., \& Spielberger, C. D. (2004). The measurement of perceptual curiosity. Personality and Individual Differences, 36(5), 1127-1141. https://doi.org/10.1016/S0191-8869(03)00205-8.

Compton, W. C., \& Hoffman, E. (2019). Positive psychology: the science of happiness and flourishing. Newbury Park: Sage Publications.

Evans, K. R., McFarland, R. G., Dietz, B., \& Jaramillo, F. (2012). Advancing sales performance research: A focus on five underresearched topic areas. Journal of Personal Selling \& Sales Management, 32(1), 89-105. https://doi.org/10.2753/PSS0885-3134320108.

Ferrando, P. J., \& Lorenzo-Seva, U. (2018). Assessing the quality and appropriateness of factor solutions and factor score estimates in exploratory item factor analysis. Educational and Psychological Measurement, 78(5), 762-780. https://doi.org/10.1177/0013164417719308.

Franklin, D., \& Marshall, R. (2019). Adding co-creation as an antecedent condition leading to trust in business-to-business relationships. Industrial Marketing Management, 77, 170181. https://doi.org/10.1016/j.indmarman.2018.10.002.

Griffin, M. A., Neal, A., \& Parker, S. K. (2007). A new model of work role performance: Positive behavior in uncertain and interdependent contexts. Academy of management journal, 50(2), 327-347. https://doi.org/10.5465/amj.2007.24634438.

Gross, M. E., Zedelius, C. M., \& Schooler, J. W. (2020). Cultivating an understanding of curiosity as a seed for creativity. Current Opinion in Behavioral Sciences, 35, 77-82. https://doi.org/10.1016/j.cobeha.2020.07.015.

Hair, J. F., Black, W. C., Babin, B. J., Anderson, R. E., \& Tatham, R. L. (2009). Análise multivariada de dados. Porto Alegre: Bookman editora.

Hassan, M. M., Bashir, S., \& Mussel, P. (2015). Personality, learning, and the mediating role of epistemic curiosity: A case of continuing education in medical physicians. Learning and Individual Differences, 42, 83-89. https://doi.org/10.1016/j.lindif.2015.07.018.

Herjanto, H., \& Franklin, D. (2019). Investigating salesperson performance factors: A systematic review of the literature on the characteristics of effective salespersons. Australasian Marketing Journal (AMJ), 27(2), 104-112. https://doi.org/10.1016/j.ausmj.2018.12.001.

Heggestad, E. D., \& Gordon, H. L. (2008). An argument for context-specific personality assessments. Industrial and Organizational Psychology, 1(3), 320-322. 10.1111/j.17549434.2008.00056.x.

Hurley, A. E., Scandura, T. A., Schriesheim, C. A., Brannick, M. T., Seers, A., Vandenberg, R. J., \& Williams, L. J. (1997). Exploratory and confirmatory factor analysis: Guidelines, 
issues, and alternatives. Journal of Organizational Behavior, 667-683. http://www.jstor.org/stable/3100253.

Kotze, M. (2018). How job resources and personal resources influence work engagement and burnout. African Journal of Economic and Management Studies. https://doi.org/10.1108/AJEMS-05-2017-0096.

Litman, J. A. (2008). Interest and deprivation factors of epistemic curiosity. Personality and Individual Differences, 44(7), 1585-1595. https://doi.org/10.1016/j.paid.2008.01.014.

Loewenstein, G. (1994). The psychology of curiosity: A review and reinterpretation. Psychological Bulletin, 116(1), 75. http://www.andrew.cmu.edu/user/gl20/GeorgeLoewenstein/Papers_files/pdf/Psychof Curiosity.pdf.

Lorenzo-Seva, U., \& Ferrando, P. J. (2013). Factor 9.2: A comprehensive program for fitting exploratory and semiconfirmatory factor analysis and IRT models. Applied Psychological Measurement. https://doi.org/10.1177/0146621613487794.

Luthans, F., \& Youssef, C. M. (2007). Emerging positive organizational behavior. Journal of Management, 33(3), 321-349. https://doi.org/10.1177/0149206307300814.

McDonald, R. P. (2013). Test theory: a unified treatment. Londres: Psychology press.

Mussel, P. (2010). Epistemic curiosity and related constructs: Lacking evidence of discriminant validity. Personality and Individual Differences, 49(5), 506-510. https://doi.org/10.1016/j.paid.2010.05.014.

Mussel, P., Spengler, M., Litman, J. A., \& Schuler, H. (2011). Development and validation of the German work-related curiosity scale. European Journal of Psychological Assessment. https://doi.org/10.1027/1015-5759/a000098.

Mussel, P. (2013). Introducing the construct curiosity for predicting job performance. Journal of Organizational Behavior, 34(4), 453-472. https://doi.org/10.1002/job.1809.

Ornstein, M. (2013). A companion to survey research. Londres: Sage Publications.

Peters, R. A. (1978). Effects of anxiety, curiosity, and perceived instructor threat on student verbal behavior in the college classroom. Journal of Educational Psychology, 70(3), 388-395. https://doi.org/10.1037/0022-0663.70.3.388.

Pulakos, E. D., Arad, S., Donovan, M. A., \& Plamondon, K. E. (2000). Adaptability in the workplace: Development of a taxonomy of adaptive performance. Journal of Applied Psychology, 85(4), 612-624. https://doi.org/10.1037/0021-9010.85.4.612.

Rosa, H. (2013). Social acceleration: a new theory of modernity. New York: Columbia University Press. 
Reeve, J. (2014). Understanding motivation and emotion. New Jersey: John Wiley \& Sons.

Seaton, C. L., \& Beaumont, S. L. (2008). Individual differences in identity styles predict proactive forms of positive adjustment. Identity: An International Journal of Theory and Research, 8(3), 249-268. https://doi.org/10.1080/15283480802181891.

Seligman, M. E. (2004). Positive psychology in practice (pp. 713-731). P. A. Linley, \& S. Joseph (Eds.). Hoboken, New Jersey: Wiley.

Thompson, B. (2004). Exploratory and confirmatory factor analysis: Understanding concepts and applications. Washington: American Psychological Association.

Timmerman, M. E., \& Lorenzo-Seva, U. (2011). Dimensionality assessment of ordered polytomous items with parallel analysis. Psychological Methods, 16(2), 209. https://doi.org/10.1037/a0023353.

Van de Vijver, F., \& Hambleton, R. K. (1996). Translating tests. European Psychologist, 1(2), 89-99. https://doi.org/10.1027/1016-9040.1.2.89.

Zhang, A. L., \& Glynn, M. S. (2015). Towards a framework of a salesperson's resource facilitation and interaction. Australasian Marketing Journal (AMJ), 23(2), 124-131. https://doi.org/10.1016/j.ausmj.2015.04.007.

Zuckerman, M. (1994). Behavioral expressions and biosocial bases of sensation seeking. New York: Cambridge university press.

Wright, J. C., \& Mischel, W. (1987). A conditional approach to dispositional constructs: the local predictability of social behavior. Journal of Personality and Social Psychology, 53(6), 1159. https://doi.org/10.1037/0022-3514.53.6.1159. 